\title{
Fatores associados à prática de exercícios físicos realizados em tempos de pandemia de COVID-19
}

\section{Factors associated with praticing physical exercises in times of the COVID-19 pandemic}

\author{
Caroline dos Santos ${ }^{1}$, Diene da Silva Schlickmann ${ }^{1}$, Thalia Gama da Silva ${ }^{1}$, Eduarda da Silva \\ Limberger Castilhos ${ }^{1}$, Patrícia Molz ${ }^{1,2}$, Silvia Isabel Rech Franke ${ }^{1}$ \\ 1 - Universidade de Santa Cruz do Sul - UNISC, Santa Cruz do Sul, RS, Brasil. \\ 2 - Pontifica Universidade Católica do Rio Grande do Sul - PUCRS, Porto Alegre, RS, Brasil.
}

\section{RESUMO}

Introdução: a COVID-19 fez com que a população mundial se adequasse ao novo cenário pandêmico com a imposição do distanciamento social. Com isso, muitos hábitos de vida foram modificados, tais como a prática regular de exercícios físicos. Objetivo: verificar os fatores associados à prática de exercícios físicos realizados em casa em tempos de pandemia da COVID-19. Método: estudo transversal, realizado com 149 indivíduos de 18 a 60 anos entre abril a julho de 2020. Utilizou-se um questionário on-line contendo questões sobre dados sociodemográficos (sexo e faixa etária), de hábitos de vida e de saúde (uso de fumo, consumo de álcool,

silviafr@unisc.br presença de doença, estado de saúde, nível de estresse percebido, horas de sono e mudança de peso), questões voltadas à prática de exercícios físicos realizados em casa durante o distanciamento social (tipo, frequência, tempo, satisfação e as dificuldades encontradas), e se os indivíduos praticavam academia antes da pandemia. Os dados foram analisados pelos testes Qui-quadrado de Pearson ou Exato de Fischer, com nível de significância de $p<0,05$. Resultados: dos indivíduos avaliados, $66,0 \%$ relataram praticar exercícios físicos em casa, com maior prevalência do sexo feminino $(75,8 \%$ vs $24,2 \%$; $p=0,482)$ e de indivíduos com mais de 30 anos $(60,6 \%$ vs $39,4 \% ; \mathrm{p}=0,275$ ). Apenas a prática de exercícios realizados em academia antes do período de distanciamento social associou-se a prática de exercícios realizados em casa durante o distanciamento social $(84,8 \%$ vs $38,0 \%$; $\mathrm{p}<0,001)$. Entre os indivíduos que relataram praticar exercícios em casa, a musculação foi a atividade mais

Palavras-chave: realizada por ambos os sexos (45,3\% mulheres vs $58,3 \%$ homens). Ainda, a maioria dos indivíduos relataram se exercitar de três a quatro vezes na semana ( $41,3 \%$ mulheres vs $37,5 \%$ homens) e por mais de 30 minutos (54,7\% mulheres vs $54,2 \%$ homens) por treino. A maioria, também relatou sentir-se satisfeito com a prática de exercícios físicos em casa (53,3\% mulheres e 58,3\% homens). Conclusão: conclui-se que a maioria dos Academias de ginástica;

Estilo de vida;

COVID-19; Exercício Físico; Fatores de Risco. indivíduos avaliados estavam realizando exercícios em casa, durante o distanciamento social, o que se associou apenas à prática de academia antes do período de pandemia da COVID-19.

\begin{abstract}
Introduction: COVID-19 made the world population adapt to the new pandemic scenario with the imposition of social distancing. As a result, many lifestyle habits have been modified, such as regular physical exercise. Objective: to verify the factors associated with practicing physical exercises at home during the period of social distance due to the COVID-19 pandemic. Method: cross-descriptive study carried out with 149 individuals from 18 to 60 years, between April and July 2020. An online questionnaire was used containing questions about sociodemographic (sex and age group), life and health habits data (use of tobacco, alcohol consumption, presence of illness, health status, perceived stress level, hours of sleep, and weight change), questions related to the practice of physical exercises at home during social distancing (type, frequency, time, satisfaction and difficulties encountered), and whether individuals used to go to the gym before the pandemic. Statistical analyses were performed using Pearson's Chi-square or Fischer's Exact test with a significance level of $p<0.05$. Results: of the individuals evaluated, $66.0 \%$ reported practicing physical exercises at home, with a higher prevalence of females $(75.8 \%$ vs. $24.2 \%$; $\mathrm{p}=0.482)$ and individuals over 30 years old $(60.6 \%$ vs. $39.4 \% ; \mathrm{p}=0.275)$. Only the practice of exercises in a gym before the period the pandemic was associated with the practice of exercises at home during social distancing $(84.8 \%$ vs. $38.0 \%$; $\mathrm{p}<0.001)$. Among individuals who reported exercising at

Keywords: home, weight training was the activity most performed by both sexes ( $45.3 \%$ women vs. $58.3 \%$ men). In addition, most individuals reported exercising three to four times a week ( $41.3 \%$ women vs. $37.5 \%$ men) and for more than 30 minutes $(54.7 \%$ women vs. $54.2 \%$ men) per workout. The majority also reported feeling satisfied with exercising at home (53.3\% women vs. $58.3 \%$ men). Conclusion: most of the individuals evaluated had been exercising at home, during social distancing, which was associated only with going to a gym before the COVID-19 pandemic. Fitness Centers; Life Style; COVID-19; Exercise; 


\section{INTRODUÇÃO}

Em dezembro de 2019, o mundo entrou em alerta devido a descoberta do novo cornonavírus (Sars$\mathrm{CoV}-2)$, surgindo uma epidemia global. ${ }^{1}$ Com o intuito de evitar a propagação do vírus, o Brasil e o resto do mundo adotaram diversas medidas preventivas, tais como a restrição social. ${ }^{2,3}$ No âmbito do Estado do Rio Grande do Sul/Brasil, foi instituído o Sistema de Distanciamento Controlado, que determinou o distanciamento social como uma das medidas de prevenção e de enfrentamento à pandemia. ${ }^{4}$

O distanciamento social proporcionou diversas mudanças no estilo de vida das pessoas, principalmente entre aquelas adeptas da prática de exercícios físicos. ${ }^{2,5,6}$ Consequentemente, diminuiuse a prática de exercícios físicos realizados ao ar livre, bem como os realizados em academias, ${ }^{7,8} \mathrm{em}$ virtude do fechamento excepcional de diversos estabelecimentos, inclusive das academias, conforme decreto do estado do Rio Grande do Sul e de outros estados do Brasil. ${ }^{4}$

Para que a população se mantivesse ativa, a fim de evitar as consequências negativas para o bem estar físico e mental durante o período de pandemia, o Ministério da Saúde propôs recomendações para a realização de exercícios em casa durante o distanciamento social. ${ }^{9}$ Com isso, novos hábitos foram incentivados como a prática de exercícios ao ar livre, o deslocamento ativo, além de alongamentos e exercícios de fortalecimento, a fim de manter o corpo em constante movimento. ${ }^{6,10,11}$ Estas estratégias estão relacionadas às evidências anteriores, nos quais apontam que a prática de exercícios físicos influencia diretamente na saúde das pessoas, auxiliando na manutenção da saúde mental e no controle das descompensações de comorbidades, que são um dos principais riscos para o desenvolvimento da COVID-19..$^{12-14}$

Baseados na observação de que as medidas restritivas adotadas com o distanciamento social para conter o número de mortes por COVID-19 estão afetando drasticamente o nível de atividade física. Mudanças no estilo de vida ativo neste período são inevitáveis e podem impactar diretamente na manutenção da saúde. Portanto, torna-se importante compreender como os fatores comportamentais e psicossociais dos indivíduos estão interferindo no seu estado de saúde e poder lançar luzes sobre questões relevantes para futuras estratégias para reduzir a inatividade física, estimulando a prática de exercícios físicos em casa. Sendo assim, o objetivo do presente estudo foi verificar os fatores associados à prática de exercícios físicos realizados em casa em tempos de pandemia da COVID-19.

\section{MÉTODO}

Estudo transversal, aprovado pelo Comitê de Ética em Pesquisa da Universidade de Santa Cruz do Sul, sob parecer $n^{\circ} 20.20 .170$, seguindo todas as diretrizes estabelecidas na Declaração de Helsinque e a resolução 466/2012 do Conselho Nacional de Saúde do Brasil. Foram avaliados 149 indivíduos, sendo 112 do sexo feminino e 37 do sexo masculino com idade entre 18 e 60 anos, no período de abril a julho de 2020, durante o período do distanciamento social controlado, imposto pelo governo do estado do Rio Grande do Sul, devido à pandemia da COVID-19. A amostragem foi por conveniência e incluiu-se no estudo, indivíduos entre 18 e 60 anos, de ambos os sexos e que consentiram com o termo de consentimento livre e esclarecido (TCLE) de forma on-line. Foram excluídos do estudo indivíduos que não preencheram corretamente o questionário on-line.

Para a coleta dos dados estruturou-se um questionário on-line na plataforma Google Forms, que continham questões sobre dados sociodemográficos (sexo e faixa etária), de hábitos de vida e de saúde (uso de fumo, consumo de álcool, presença de doença, estado de saúde, nível de estresse percebido, horas de sono e mudança de peso), questões voltadas à prática de exercícios físicos realizados em casa durante o distanciamento social (tipo, frequência, tempo, satisfação e as dificuldades encontradas), e se os indivíduos praticavam academia antes da pandemia. O instrumento foi disponibilizado por meio de aplicativos de mensagens, e-mails e redes sociais.

Para análise dos dados, utilizou-se o programa estatístico Statistical Package for the Social Sciences (SPSS versão 23.0). Utilizou-se o teste de Quiquadrado de Pearson ou Exato de Fischer para avaliar as associações entre as variáveis, considerando $\mathrm{p}<0,05$ como nível de significância.

\section{RESULTADOS}

Do total de participantes do estudo, $66,0 \%$ relataram praticar exercícios físicos em casa, durante o período de distanciamento controlado. Como mostrado 
na tabela 1 , a realização de exercícios físicos em casa foi mais relatada pelas mulheres $(75,8 \%$ vs $24,2 \%$; $\mathrm{p}=0,482$ ), bem como pelos indivíduos com mais de 30 anos $(60,6 \%$ vs 39,4\%; $\mathrm{p}=0,275)$. Além disso, a maioria dos indivíduos avaliados não tinha o hábito de fumar $(94,0 \%$ vs 97,0\%; $\mathrm{p}=0,322)$. Contudo, a maioria dos indivíduos que relatou praticar exercícios físicos, ingeriam álcool uma vez na semana $(39,4 \%)$, enquanto a maior parte dos indivíduos que não praticavam exercícios físicos, relataram não ingerir álcool $(38,0 \%$; $=0,203)$.
Embora a maioria dos indivíduos tenha relatado não apresentar doença $(81,9 \%)$, os indivíduos que apresentavam alguma doença relataram, com maior prevalência, realizar exercícios em casa $(19,2 \%$ vs $16,0 \% ; \mathrm{p}=0,406)$. Além disso, tanto os indivíduos que não praticavam exercícios como os que praticavam, na sua maioria, relataram apresentar estado de saúde excelente ou bom (70,0\% vs 70,7\%; $\mathrm{p}=0,537)$ e que às vezes sentiam-se estressados, mas conseguiam viver razoavelmente bem $(64,0 \%$ vs $62,6 \% ; p=0,534$, tabela 1$)$.

Tabela 1 - Características sociodemográficas e de hábitos de vida entre indivíduos que praticavam ou não exercícios físicos realizados em casa, durante o distanciamento social devido à Covid-19.

\begin{tabular}{|c|c|c|c|c|}
\hline \multirow[b]{2}{*}{ Variáveis } & \multirow{2}{*}{$\begin{array}{l}\text { Total } \\
\text { n (\%) }\end{array}$} & \multicolumn{2}{|c|}{ Exercícios físicos em casa } & \multirow[t]{2}{*}{ Valor $\mathrm{p}$} \\
\hline & & $\begin{array}{c}\text { Não } \\
\text { n }(\%)\end{array}$ & $\begin{array}{c}\text { Sim } \\
\text { n }(\%)\end{array}$ & \\
\hline Sexo & & & & 0,482 \\
\hline Feminino & $112(75,2 \%)$ & $37(74,0 \%)$ & $75(75,8 \%)$ & \\
\hline Masculino & $37(24,8 \%)$ & $13(26,0 \%)$ & $24(24,2 \%)$ & \\
\hline Faixa etária & & & & 0,275 \\
\hline$<30$ anos & $62(41,6 \%)$ & $23(46,0 \%)$ & $39(39,4 \%)$ & \\
\hline$>30$ anos & $87(58,4 \%)$ & $27(54,0 \%)$ & $60(60,6 \%)$ & \\
\hline Fumante & & & & 0,322 \\
\hline Não & $143(96,0 \%)$ & $47(94,0 \%)$ & $96(97,0 \%)$ & \\
\hline Sim & $6(4,0 \%)$ & $3(6,0 \%)$ & $3(3,0 \%)$ & \\
\hline Consumo de álcool & & & & 0,203 \\
\hline Não ingere & $49(32,9 \%)$ & $19(38,0 \%)$ & $30(30,3 \%)$ & \\
\hline 1 vez na semana & $51(34,2 \%)$ & $12(24,0 \%)$ & $39(39,4 \%)$ & \\
\hline $2-3$ vezes na semana & $35(23,5 \%)$ & $12(24,0 \%)$ & $23(23,2 \%)$ & \\
\hline$>4$ vezes na semana & $14(9,4 \%)$ & $7(14,0 \%)$ & $7(7,1 \%)$ & \\
\hline Presença de doença & & & & 0,406 \\
\hline Não & $122(81,9 \%)$ & $42(84,0 \%)$ & $80(80,8 \%)$ & \\
\hline Sim & $27(18,1 \%)$ & $8(16,0 \%)$ & $19(19,2 \%)$ & \\
\hline Estado de saúde & & & & 0,537 \\
\hline Excelente/Bom & $105(70,5 \%)$ & $35(70,0 \%)$ & $70(70,7 \%)$ & \\
\hline Regular/Ruim & $44(29,5)$ & $15(30,0 \%)$ & $29(29,3 \%)$ & \\
\hline Nível de estresse & & & & 0,534 \\
\hline $\begin{array}{l}\text { Raramente estressado, vivendo muito } \\
\text { bem }\end{array}$ & $39(26,2 \%)$ & $11(22,0 \%)$ & $28(28,3 \%)$ & \\
\hline $\begin{array}{l}\text { Às vezes estressado, vivendo } \\
\text { razoavelmente bem }\end{array}$ & $94(63,1 \%)$ & $32(64,0 \%)$ & $62(62,6 \%)$ & \\
\hline $\begin{array}{l}\text { Quase sempre ou excessivamente } \\
\text { estressado }\end{array}$ & $16(10,7 \%)$ & $7(14,0 \%)$ & $9(9,1 \%)$ & \\
\hline Sono & & & & 0,596 \\
\hline$<7$ h (sono curto/muito curto) & $45(30,2 \%)$ & $16(32,0 \%)$ & $29(29,3 \%)$ & \\
\hline $7-8 \mathrm{~h}$ (sono ideal) & $45(30,2 \%)$ & $17(34,0 \%)$ & $28(28,3 \%)$ & \\
\hline >8h (sono longo/muito longo) & $59(39,6 \%)$ & $17(34,0 \%)$ & $42(42,4 \%)$ & \\
\hline Mudança de peso & & & & 0,233 \\
\hline Não & $66(44,3 \%)$ & $20(40,0 \%)$ & $46(46,5 \%)$ & \\
\hline Sim, emagreci & $28(18,8 \%)$ & $7(14,0 \%)$ & $21(21,2 \%)$ & \\
\hline Sim, ganhei peso & $55(36,9 \%)$ & $23(46,0 \%)$ & $32(32,3 \%)$ & \\
\hline Praticava academia antes da pandemia & & & & $<0,001$ \\
\hline Não & $46(30,9 \%)$ & $31(62,0 \%)$ & $15(15,2 \%)$ & \\
\hline Sim & $103(69,1 \%)$ & $19(38,0 \%)$ & $84(84,8 \%)$ & \\
\hline
\end{tabular}


Por outro lado, $42,4 \%$ dos indivíduos que praticavam exercícios físicos relataram dormir mais de $8 \mathrm{~h}$, enquanto que os indivíduos que não praticavam exercícios dormiam mais de $7 \mathrm{~h}$ por noite $(68,0 \%$; $\mathrm{p}=0,596)$. Em relação à mudança de peso, a maioria dos indivíduos que realizava exercícios não relatou alteração de peso $(46,5 \%)$, enquanto que $46,0 \%$ dos indivíduos que não praticavam exercícios relataram ter ganhado peso durante o período de distanciamento controlado $(p=0,233)$. Além disso, a maioria dos indivíduos que realizava exercícios físicos em casa relatou frequentar a academia para praticar exercícios físicos antes do período de distanciamento controlado
$(84,8 \%$ vs $38,0 \% ; \mathrm{p}<0,001)$.

Em relação à prática de exercícios em casa (figura 1), a atividade mais realizada por ambos os sexos foi a musculação (45,3\% mulheres vs 58,3\% homens). Além disso, os homens relataram também praticar treino funcional $(29,2 \%)$; e as mulheres relataram praticar exercícios aeróbicos $(28,0 \%)$ e treino funcional $(26,7 \%)$. Ambos os sexos também relaram, na sua maioria, realizar exercícios físicos em casa de 3 a 4 vezes na semana (41,3\% mulheres vs $37,5 \%$ homens) e por mais de 30 minutos $(54,7 \%$ mulheres vs 54,2\% homens) por treino.

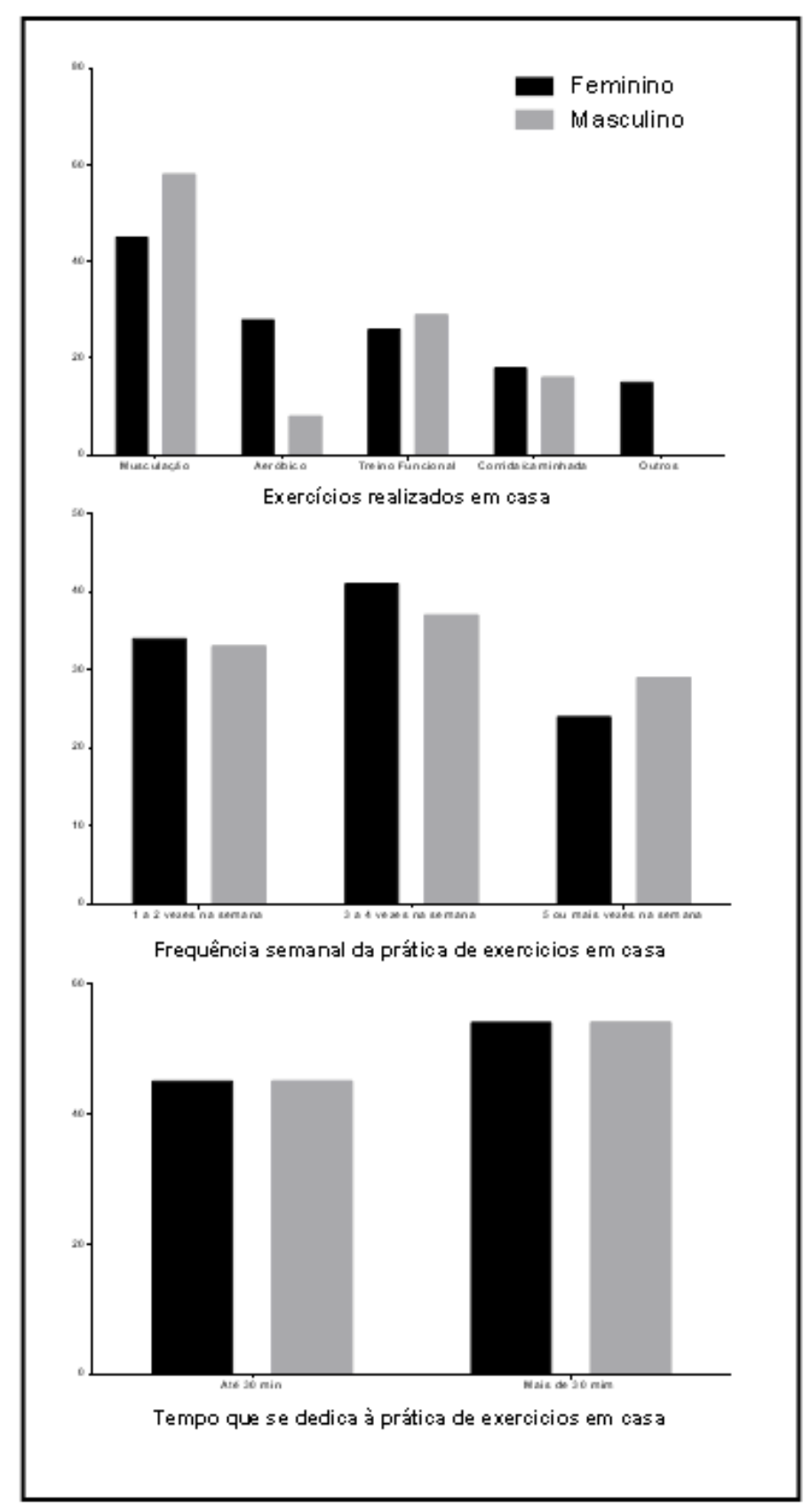

Figura 2 - Satisfação e as dificuldades encontradas para a prática de exercícios físicos realizados em casa, por homens e mulheres, durante o distanciamento social devido à Covid-19. 
Figura 1 - Tipo, frequência e tempo da prática de exercícios físicos realizados em casa, por homens e mulheres, durante o distanciamento social devido à Covid-19.

Quanto à satisfação com a prática de exercícios físicos em casa (figura 2), 53,3\% das mulheres e $58,3 \%$ dos homens relataram sentir-se satisfeitos. Entretanto, as principais dificuldades em realizar exercícios em casa citadas pelas mulheres foram a falta de motivação/disciplina $(22,7 \%)$ e de espaço e/ou equipamentos (20,0\%). Já, entre os homens, a maior dificuldade encontrada foi a falta de espaço e/ ou equipamentos $(41,7 \%)$.

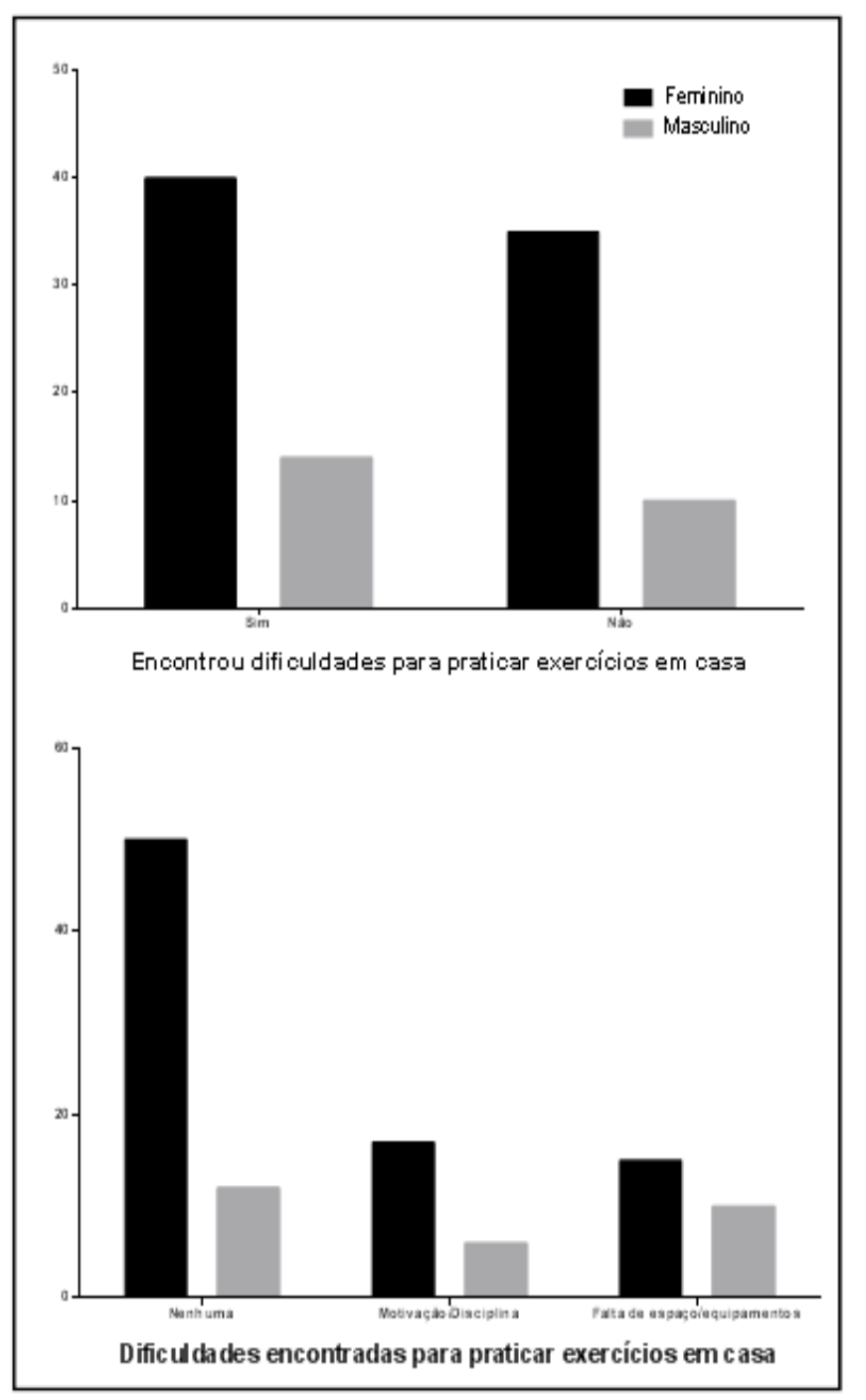

Figura 2 - Satisfação e as dificuldades encontradas para a prática de exercícios físicos realizados em casa, por homens e mulheres, durante o distanciamento social devido à Covid-19.

\section{DISCUSSÃO}

No presente estudo, a prática de exercícios realizados em casa durante o distanciamento social foi relatada por $66,0 \%$ dos indivíduos avaliados e associou-se à prática de exercícios realizados em academia antes do período de distanciamento social. Além disso, a maioria dos indivíduos que relataram praticar exercícios em casa, praticava musculação, de três a quatro vezes na semana e por mais de 30 minutos, bem como relatou sentir-se satisfeito com a prática de exercícios físicos em casa. Esses resultados é decorrência do distanciamento social devido à COVID-19, pois a população mundial precisou se adequar ao novo cenário pandêmico, substituindo os locais da prática de exercícios físicos, que antes eram em clubes, academias e até mesmo exercícios ao ar livre, pelos exercícios físicos realizados dentro de casa. ${ }^{11}$ Apesar de alguns estudos demonstrarem diminuição na prática de exercícios físicos durante este período, ${ }^{8,12}$ nossos resultados mostraram alta prevalência da prática de exercício físico em casa, durante o distanciamento social. Prevalência inferior ao encontrado no presente estudo, foi observado por outro estudo que avaliou a percepção da população brasileira sobre o isolamento social durante a pandemia de COVID-19, no qual observaram que $40,0 \%$ dos indivíduos praticaram algum tipo de exercício físico. ${ }^{2}$

No presente estudo, as mulheres, bem como os indivíduos acima de 30 anos foram os que mais praticaram exercícios físicos em casa, mostrando que estes indivíduos são os mais preocupados em se manter fisicamente ativos. O estudo de Malta et al. ${ }^{6}$ verificou uma diminuição na prática de exercícios físicos devido à pandemia. Embora os autores tenham verificado redução da prática de exercícios físicos em ambos os sexos, os homens mantiveram proporção maior na realização exercícios físicos em comparação às mulheres $(14,0 \%$ vs $10,3 \%)$. Entretanto, similar aos nosso, Malta et al. ${ }^{6}$ também verificaram uma maior prevalência da realização de exercícios físicos pelos indivíduos maiores de 30 anos $(49,0 \%)$, mas não determinaram se esses exercícios eram realizados em casa. Este cenário mostra que o distanciamento social ocasionou restrições que interferiram na realização de atividades cotidianas, entre elas, a prática de exercícios físicos, fazendo com que as pessoas fossem orientadas a permanecerem em casa para evitar a propagação do vírus. ${ }^{15}$ Entretanto, a população foi incentivada a dar continuidade a prática de exercícios físicos em casa, 
para manter o nível de condicionamento físico, bem como manter e/ou melhorar seu estado de saúde. ${ }^{16}$

O tabaco e o consumo excessivo de álcool também são considerados importantes fatores de risco para as complicações das infecções ocasionadas pelo Coronavírus (Sars-cov-2). 1,17 Além disso, estudos indicam que o consumo de álcool isoladamente, bem como associado ao tabaco, são comportamentos de risco que tem aumento durante o distanciamento social. ${ }^{6,8,18}$ No presente estudo, independente da prática ou não de exercícios em casa, a prevalência de consumo de tabaco foi de $4,0 \%$, enquanto que a maioria dos indivíduos avaliados referiram ingerir álcool 1 vez na semana (34,2\%). Interessantemente, estes resultados são similares a estudos que observaram indivíduos praticantes de exercícios físicos antes da pandemia, ${ }^{19,20}$ indicando que os praticantes de exercícios de forma recreacional ingerem álcool frequentemente, mas não consomem tabaco.

A literatura tem evidenciado os benefícios da prática regular de exercícios físicos como uma importante estratégia para melhorar a saúde dos indivíduos nesse período de distanciamento social. ${ }^{11,21}$ Assim, a prática de exercícios físicos tem sido mostrada como aliada de um estilo de vida mais saudável, bem como parece atenuar os riscos de internação hospitalar por COVID-19. 2,22 No presente estudo, a maioria dos indivíduos que relatou apresentar algum tipo de doença, estavam praticando exercícios em casa. Evidências tem mostrado que o exercício físico crônico apresenta um efeito positivo sobre a promoção de um ambiente anti-inflamatório, essencial para o combate de inúmeras comorbidades, que estão entre os principais fatores de risco para o desenvolvimento da COVID-19..$^{12,14}$

A interrupção de uma vida normal e as ordens de permanência em casa promoveram consequências que impactaram negativamente no bem-estar e na saúde mental dos indivíduos. ${ }^{23}$ Tem se observado que o distanciamento social pode estar associado a piora do estado de saúde, maiores níveis de estresse, assim como alteração na rotina do sono dos indivíduos e pode promover mudanças no peso corporal., ${ }^{2}$ No presente estudo, a maioria dos indivíduos que praticavam ou não exercícios físicos em casa relataram estado de saúde excelente/bom, bem como que estavam às vezes estressado, mas estavam vivendo razoavelmente bem. Além disso, os indivíduos que praticavam exercícios em casa relataram com maior prevalência do que os que não estavam realizando exercícios, que raramente estavam estressados e estavam vivendo muito bem $(28,3 \%$ vs $22,0 \%)$. Nossos achados corroboram com um estudo brasileiro que, ao avaliar aspectos comportamentais e sociais durante o distanciamento social, verificou que os indivíduos mais estressados estavam realizando menos atividades físicas. ${ }^{7}$ Evidências também têm mostrado o quanto a prática de exercícios pode auxiliar na melhoria do bem-estar, principalmente neste período de incertezas. ${ }^{12,14}$

O sono desempenha papel importante na saúde mental e física dos indivíduos. Além disso, a qualidade e horas de sono adequados são essenciais para manutenção da saúde também em tempos de pandemia da COVID-19. ${ }^{24}$ Em nosso estudo, a maioria dos indivíduos apresentava sono longo ou muito longo $(39,6 \%)$, possivelmente estando relacionado com as alterações de rotina pessoal, devido ao distanciamento social. ${ }^{25}$ Contudo, Morin et al. ${ }^{24}$ sugerem priorizar pelo menos de 7 a 8 horas de sono durante a pandemia da Covid-19. Ainda, no presente estudo, observamos que os indivíduos que estavam praticando exercícios em casa apresentaram maior prevalência de sono ideal comparado aos indivíduos que não estavam praticando exercícios. Resultados similares foram encontrados por Bezerra et al. ${ }^{2}$, no qual verificaram que os indivíduos $(50,3 \%)$ que praticavam atividade física revelaram maior normalidade no sono.

Muitos indivíduos também têm recorrido a prática de exercícios físicos para promover mudanças na composição corporal durante o distanciamento social. ${ }^{13}$ Nosso estudo mostrou que indivíduos que realizavam exercícios físicos em casa tiveram maior redução de peso corporal $(21,1 \%)$, assim como observou-se um maior ganho de peso $(46,0 \%)$ entre os que não praticaram exercícios físicos. Esses dados enfatizam o aumento na frequência dos comportamentos de risco durante a pandemia, no qual a restrição social pode influenciar na ingestão e o gasto de energia, afetando o balanço energético e contribuindo para o ganho de peso. ${ }^{3}$ Ainda, este comportamento é preocupante e pode resultar em danos à saúde, bem como no aumento na ocorrência de doenças crônicas não transmissíveis. ${ }^{6}$

Manter a prática regular de exercícios físicos, mesmo que no ambiente doméstico, é uma importante estratégia para manter e/ou melhorar à saúde durante a pandemia ocasionada pela COVID-19. ${ }^{16}$ No presente estudo, observou-se que $84,8 \%$ dos indivíduos que realizavam exercícios físicos em casa, frequentavam a academia anteriormente à pandemia. 
Além disso, o estudo de Malta et al. ${ }^{6}$ apontou que a prática de atividade física apresentou mudanças devido à pandemia no Brasil, promovendo redução significativa. Este comportamento também poder ser observado no presente estudo, uma vez que 38,0\% dos indivíduos que relataram frequentar a academia anteriormente a pandemia pararam de se exercitar.

Em se tratando de recomendações para a prática de exercícios em casa, França et al. ${ }^{10}$ sugeriram estratégias para se manter fisicamente ativo e seguro dentro de casa, dentre as quais estão exercícios de alongamento e academias online. No presente estudo, o exercício mais relatado, tanto por homens, quanto por mulheres foi a musculação. Em parte, este resultado está associado com o aumento do número de academias e de profissionais de Educação Física autônomos que têm ministrado aulas remotas, ofertando a prescrição de exercícios físicos para serem realizados em casa. ${ }^{10,26}$ Além disso, os homens também relataram praticar treinamento funcional, enquanto as mulheres, relataram praticar exercícios aeróbicos e treinamento funcional. Segundo Raiol et al. ${ }^{27}$, exercícios aeróbicos podem ser adaptados a qualquer indivíduo e podem ser facilmente realizados em casa, utilizando o peso do próprio corpo. Os autores ainda relatam que, o treinamento funcional também é um exercício que pode ser realizado em casa, utilizando materiais, objetos encontrados no ambiente doméstico e até mesmo ser realizado com o peso corporal.

Recentemente a World Health Organization $(\mathrm{WHO})^{15}$ publicou as novas diretrizes para a prática de atividade física, sugerindo de 150 a 300 minutos por semana de atividade física aeróbica de intensidade moderada e duas sessões por semana de treinamento de força muscular para adultos. No presente estudo, observou-se que a maioria dos indivíduos que relataram praticar exercícios físicos exercitavam-se entre 3 a 4 vezes na semana e por mais de 30 minutos por treino. Estes resultados apontam que a maioria dos indivíduos do presente estudo tem cumprido as recomendações da WHO.

A maioria das mulheres e dos homens relataram não apresentar dificuldades para realizar exercícios em casa. Contudo, entre as principais dificuldades relatadas pelas mulheres foram a falta de motivação/ disciplina e a falta de espaço/equipamentos, enquanto os homens relataram como dificuldade a falta de espaço/equipamentos.
Uma limitação do presente estudo é que a amostra não é representativa fiel da população, devido à baixa adesão dos pesquisados. Isto pode não ser condizente com a realidade dos mesmos.

\section{CONCLUSÃO}

A maioria dos indivíduos avaliados relatou praticar exercícios físicos em casa, sendo mais realizado pelas mulheres e pelos indivíduos com mais de 30 anos. Apenas a realização de exercícios em casa associou-se à prática de exercícios em academia antes do período de distanciamento social.

Além disso, a atividade mais realizada entre os indivíduos que relataram praticar exercícios em casa, independente do sexo, foi a musculação. A maioria dos indivíduos também relatou se exercitar de 3 a 4 vezes na semana e por mais de 30 minutos por treino. Mesmo que a maioria dos indivíduos que praticavam exercícios fiscos em casa relataram que se sentiam satisfeitos, a motivação/disciplina e a falta de espaço/equipamentos foram as maiores dificuldade encontradas para a realização dos exercícios.

\section{Agradecimentos}

Agradecemos os bolsistas de pesquisa Fapergs, $\mathrm{CNPq}$, Capes e PUIC/Unisc do Laboratório de Nutrição Experimental da UNISC pela divulgação do questionário e a Nutricionista Morgana Tonett pelo auxílio na construção do instrumento utilizado na coleta dos dados dessa pesquisa.

\section{REFERÊNCIAS}

1. Silva AL, Moreira JC, Martins SR. COVID-19 and smoking: a high-risk association. Cad Saúde Pública 2020;36:e00072020. doi: https://doi.org/10.1590/0102-311X00072020

2. Bezerra AC, Silva CE, Soares FR, Silva JA. Fatores associados ao comportamento da população durante o isolamento social na pandemia de COVID-19. Ciênc Saúde Colet 2020; 25(suppl 1). doi: https://doi.org/10.1590/1413-81232020256.1.10792020

3. Bhutani S, Cooper JA. COVID-19 related home confinement in adults: weight gain risks and opportunities. Obesity. 2020. doi: https://dx.doi.org/10.1002\%2Foby.22904

4. Estado do Rio Grande do Sul. Decreto Estadual no 55.240, dia 10 de maio de 2020. Institui o Sistema de Distanciamento Controlado para fins de prevenção e de enfrentamento à epidemia causada pelo novo Coronavírus (COVID-19) no âmbito do Estado do Rio Grande do Sul, reitera a declaração de estado de calamidade pública em todo o território estadual e dá outras providências. Rio Grande do Sul, 2020.

5. Hamer M, Kivimäki M, Gale CR, Batty GD. Lifestyle risk factors, inflammatory mechanisms, and COVID-19 
hospitalization: A community-based cohort study of 387,109 adults in UK. Brain Behav Immun. 2020 Jul; 87: 184-187. doi: https://dx.doi.org/10.1016\%2Fj.bbi.2020.05.059

6. Malta DC, Szwarcwald CL, Barros MB, Gomes CS, Machado ÍE, Souza Júnior PR, Romero DE, Lima MG, Damacena GN, Pina MD, Freitas MI. A pandemia da COVID-19 e as mudanças no estilo de vida dos brasileiros adultos: um estudo transversal, 2020. Epidemiol Serv Saúde. 2020;29:e2020407. doi: https:// doi.org/10.1590/S1679-49742020000400026

7. Filgueiras A, Stults-Kolehmainen M. The Relationship Between Behavioural and Psychosocial Factors Among Brazilians in Quarantine Due to COVID-19. SSRN Electron J 2020. doi: http://dx.doi.org/10.2139/ssrn.3566245

8. Peçanha T, Goessler KF, Roschel H, Gualano B. Social isolation during the COVID-19 pandemic can increase physical inactivity and the global burden of cardiovascular disease. American Am J Physiol Heart Circ Physiol 2020;318(6):H1441-H1446. doi: https://doi.org/10.1152/ajpheart.00268.2020

9. Brasil. Ministério da Saúde. Como fica a prática de atividade física durante a pandemia de Coronavírus?. Ministério da Saúde, 20 de maio de 2020. Disponível em: https://saudebrasil.saude. gov.br/eu-quero-me-exercitar-mais/como-fica-a-pratica-deatividade-fisica-durante-a-pandemia-de-coronavirus

10. França EF, Miyake GM, da Silva Júnior JP, Matsudo VK, Martins RÁ, Nascimento FD. COVID-19: Estratégias para se manter fisicamente ativo e seguro dentro de casa. InterAm J Med Health 2020;3:e202003034. doi: https://doi.org/10.31005/iajmh. v3i0.122

11. Schwendinger F, Pocecco E. Counteracting Physical Inactivity during the COVID-19 Pandemic: Evidence-Based Recommendations for Home-Based Exercise. Int J Environ Res Public Health 2020;17(11):3909. doi: https://dx.doi. org/10.3390\%2Fijerph17113909

12. Meyer J, McDowell C, Lansing J, Brower C, Smith L, Tully M, Herring M. Changes in physical activity and sedentary behavior in response to COVID-19 and their associations with mental health in 3052 US adults. Int J Environ Res Public Health 2020;17(18):6469. doi: https://dx.doi. org $/ 10.3390 \% 2$ Fijerph 17186469

13. Swift DL, McGee JE, Earnest CP, Carlisle E, Nygard M, Johannsen NM. The Effects of Exercise and Physical Activity on Weight Loss and Maintenance. Prog Cardiovasc Dis 2018;61(2):206-213. doi: https://dx.doi.org/10.1016/j. pcad.2018.07.014

14. Yamada AK, Pólis LO. COVID-19 e sistema imune: qual o papel do exercício físico e recomendações práticas?. Saúde em Revista. 2020;20(52):57-66. doi: https://doi.org/10.15600/22381244/sr.v20n52p57-66

15. World Health Organization. Coronavirus disease (COVID-19) advice for the public. 2020. [atualizado em 8 de dezembro de 2020] Disponível em: https://www.who.int/emergencies/ diseases/novel-coronavirus-2019/advice-for-public

16. Chen P, Mao L, Nassis GP, Harmer P, Ainsworth B, Li F. Returning Chinese school-aged children and adolescents to physical activity in the wake of COVID-19: Actions and precautions. J Sport Health Sci 2020;9(4):322-324. doi: https:// dx.doi.org/10.1016\%2Fj.jshs.2020.04.003

17. GarciaLP, SanchezZM. Consumo deálcooldurantea pandemia da COVID-19: uma reflexão necessária para o enfrentamento da situação. Cad Saúde Pública 2020;36:e00124520. doi: https:// doi.org/10.1590/0102-311X00124520

18. Wang C, Pan R, Wan X, Tan Y, Xu L, McIntyre RS, Choo FN, Tran B, Ho R, Sharma VK, Ho C. A longitudinal study on the mental health of general population during the COVID-19 epidemic in China. Brain Behav Immun 2020;87:40-48. doi: https://doi.org/10.1016/j.bbi.2020.04.028

19. De oliveira FY, et al. Uso de suplementação e composição corporal de praticantes de musculação na cidade de Itaqui-RS. Revista Brasileira De Nutrição Esportiva, v. 11, n. 62, p. $192-$ 201, 2017.

20. Silva FQ et al. Consumo de suplementos nutricionais em praticantes de atividade física de uma academia do oeste da Bahia. Revista Científica da Faminas, v. 14, n. 1, 2019.

21. Pitanga FJ, Beck CC, Pitanga CP. Inatividade física, obesidade e COVID-19: perspectivas entre múltiplas pandemias. Bras Ativ Fís Saúde 2020;25:1-4. doi: https://doi.org/10.12820/ rbafs. $25 \mathrm{e} 0114$

22. Torales J, O’Higgins M, Castaldelli-Maia JM, Ventriglio A. The outbreak of COVID-19 coronavirus and its impact on global mental health. Int J Soc Psychiatry 2020;66(4):317-320. doi: https://doi.org/10.1177/0020764020915212

23. Brooks SK, Webster RK, Smith LE, Woodland L, Wessely S, Greenberg N, Rubin GJ. The psychological impact of quarantine and how to reduce it: rapid review of the evidence. Lancet 2020;395(10227):912-920.doi: https://doi.org/10.1016/s01406736(20)30460-8

24. Morin CM, Carrier J, Bastien C, Godbout R. Sleep and circadian rhythm in response to the COVID-19 pandemic. Can J Public Health 2020;111(5):654-657. doi: https://dx.doi.org/10.1 7269\%2Fs41997-020-00382-7

25. Blume C, Schmidt MH, Cajochen C. Effects of the COVID-19 lockdown on human sleep and rest-activity rhythms. Curr Biol. 2020;30(14):R795-R797. doi: https://dx.doi.org/10.1016\%2Fj. cub.2020.06.021

26. Miguel H. Impactos da Covid-19 sobre o Personal Trainer. InterAm J Med Health,. 2020;3.

27. Raiol RA, Sampaio AM, Fernandes ID. Alternativas para a prática de exercícios físicos durante a pandemia da COVID-19 e distanciamento social. Braz J Hea Rev 2020;3(4):10232-42. doi: https://doi.org/10.34119/bjhrv3n4-247

Recebido em: 18/12/2020

Aceito em:18/03/2021

Como citar: SANTOS, Caroline dos et al. Fatores associados à prática de exercícios físicos realizados em tempos de pandemia de Covid-19. Revista Interdisciplinar de Promoção da Saúde, Santa Cruz do Sul, v. 3, n. 4, out 2020. ISSN 2595-3664. Disponível em: <https://online.unisc.br/seer/index.php/ripsunisc/article/view/16105>. Acesso em: 01 out 2020. doi:https://doi.org/10.17058/ rips.v3i4.16105 